\title{
Relative velocity distribution of inertial particles in turbulence: A numerical study
}

\author{
Vincent E. Perrin and Harm J. J. Jonker \\ Delft University of Technology, Delft, The Netherlands \\ (Received 12 December 2014; revised manuscript received 1 April 2015; published 30 October 2015)
}

\begin{abstract}
The distribution of relative velocities between particles provides invaluable information on the rates and characteristics of particle collisions. We show that the theoretical model of Gustavsson and Mehlig [K. Gustavsson and B. Mehlig, J. Turbul. 15, 34 (2014)], within its anticipated limits of validity, can predict the joint probability density function of relative velocities and separations of identical inertial particles in isotropic turbulent flows with remarkable accuracy. We also quantify the validity range of the model. The model matches two limits (or two types) of relative motion between particles: one where pair diffusion dominates (i.e., large coherence between particle motion) and one where caustics dominate (i.e., large velocity differences between particles at small separations). By using direct numerical simulation combined with Lagrangian particle tracking, we assess the model prediction in homogeneous and isotropic turbulence. We demonstrate that, when sufficient caustics are present at a given separation and the particle response time is significantly smaller than the integral time scales of the flow, the distribution exhibits the same universal power-law form dictated by the correlation dimension as predicted by the model of Gustavsson and Mehlig. In agreement with the model, no strong dependency on the Taylor-based Reynolds number is observed.
\end{abstract}

DOI: 10.1103/PhysRevE.92.043022

PACS number(s): 47.55.Kf, 92.60.hk, 47.27.Jv

\section{INTRODUCTION}

Turbulence is a phenomenological field of study, where analytically based predictions are very rare. The four-fifths law [1] is one of the few exceptions where predictions can be made from first principles. In more complex situations, such as suspended particles in turbulent flows, the nature of turbulence makes it difficult to make analytically based quantitative predictions of their dynamics, let alone the occurrence of particle collisions. The idea about turbulenceparticle interactions is that turbulence may significantly affect the particle dynamics and increase the collision probability. While analytically predicting nontrivial quantities such as the collision rate remains problematic, the collision rate of particles suspended in a turbulent flow is of interest in many research areas, such as rain formation in clouds [2-4], turbulent spray combustion, residue deposition in rivers, agglomeration of fine powders in gas flow, air filtration equipment, fast fluidized beds, and dust grain dynamics in astrophysical environments.

Wang et al. [5] showed that the frequency at which inertial particles collide in turbulent flows is determined by two different contributions. The first contribution is the radial distribution function, which is the probability of finding particles at contact, and quantifies the nonhomogeneity of the particle distribution. The second contribution is the mean radial relative velocities of the particles. In this paper we focus on quantifying the (radial) relative velocity between particles.

The radial relative velocity between particles (also known as the collision velocities) is of crucial importance to understand not only the collision rate but also the collision characteristics. Whether the collision will be elastic, nonelastic, sticky, or such that breakup of the particles will occur depends sensitively on the speed at which the particles collide [3]. Factors influencing the relative velocities are the Stokes number St (ratio of particle relaxation time $\tau_{p}=2 \rho_{p} r^{2} /\left(9 \rho_{f} v\right)$ and the Kolmogorov time scale of the flow $\left.\tau_{\eta}=(\nu /\langle\epsilon\rangle)^{1 / 2}\right)$, the mean dissipation rate $\langle\epsilon\rangle$, and the Reynolds number $\operatorname{Re}[6-16] . v$ represents the viscosity of the carrier fluid, $\rho_{p}$ the density of the particles, and $\rho_{f}$ the density of the fluid.

The sensitive dependence on the Stokes number of the velocity at which particles impact can be attributed to the formation of caustics [17,18] (also known as the sling effect $[19,20])$. This phenomenon describes the detachment of particles from the underlying flow field, allowing them to have large relative velocities at small separation. Experimental evidence of the caustic effect has been found by Bewley et al. [21]. The collision rate of particles can therefore be decomposed into a smooth contribution due to pair diffusion similar to the tracer limit of Saffman and Turner [6] and a singular contribution due to caustics [16,18,22,23], similar to the ballistic limit of Abrahamson [7].

\section{PREDICTING THE RELATIVE VELOCITIES}

In this paper we focus on the model of Gustavsson and Mehlig [16] (from now on referred to as the GM model). Gustavsson and Mehlig [16] derive an analytically based statistical description of the distribution of relative velocities of inertial particles by matching asymptotic forms of the distribution. The GM model is unique in its capacity to make analytically based, very quantitative predictions about the distribution of relative velocities $\rho(\Delta \mathbf{v}, R)$ of neighboring identical inertial particles as a function of the separation $R$ between the particles using only the correlation dimension. This distribution is also independent of the Kubo number Ku; the Kubo number is defined as $\mathrm{Ku}=u^{\prime} \tau_{\eta} / \eta$, where $u^{\prime}$ is the root mean square of the velocity field and $\eta=\left(v^{3} /\langle\epsilon\rangle\right)^{1 / 4}$ the Kolmogorov length scale.

We now briefly introduce the GM model using the original notation. Let $R=|\Delta \mathbf{x}|$ be the magnitude of the nondimensional spatial separation vector $\Delta \mathbf{x}=\mathbf{x}_{2}-\mathbf{x}_{1}$ between a particle pair and $V=|\Delta \mathbf{v}|$ be the magnitude of the nondimensional relative velocity vector $\Delta \mathbf{v}=\mathbf{v}_{2}-\mathbf{v}_{1}$. The quantities $R$ and $V$ have been made nondimensional using the relevant time and length scales of the flow. The joint probability density function 


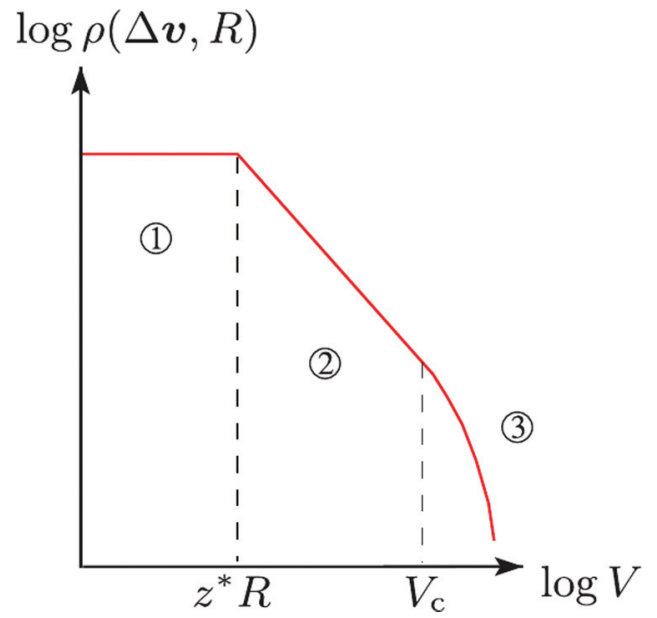

FIG. 1. (Color) Graphical representation of the three regions of the GM model, after Gustavsson and Mehlig [16].

(PDF) of the relative velocities and separations of particles suspended in randomly mixing or turbulent flow can be divided into three regimes (see Fig. 1 for a graphical representation). In regions (1) and (2) two different types of relative motion can be distinguished. In region (1) pair diffusion is dominant. Close-by particles move in a very coherent manner which implies that $V \ll R$. Since $R$ does not change much in this region, the distribution is independent of $V$. In region (2) caustics are dominant. Particles can detach from the flow field which allows large velocity differences at short separations. This implies that $V \gg R$ and that the distribution becomes independent of $R$. Clustering of the particles in combination with caustics results in a power-law distribution for the relative velocities. The exponent equals $D_{2}-2 d$, where $D_{2}$ is the phase-space correlation dimension of the particle field and $d$ the embedding spatial dimension (in our case 3 ). $z^{*}$ is the matching length scale and represents the typical value of the relative magnitude of velocity and separation between particles (i.e., $z^{*} \approx \overline{V / R}$ ). In region (3), where $V>V_{c}$, the scaling of the relative velocities loses its universal aspect and becomes system specific. $V_{c}$ is the cutoff value, above which the distribution has been set to zero as a simple assumption. Formally the joint PDF $\rho(\Delta \mathbf{v}, R)$ is given by

$$
\rho(\Delta \mathbf{v}, R) \sim R^{d-1} \begin{cases}R^{D_{2}-2 d} & \text { for region (1), } \\ \left|\Delta \mathbf{v} / z^{*}\right|^{D_{2}-2 d} & \text { for region (2), } \\ 0 & \text { for region (3), }\end{cases}
$$

where the regions (1), (2), and (3) are defined as follows:

$$
\begin{array}{ll}
\text { region (1) where } V \leqslant z^{*} R, & R \leqslant 1, \text { and } V \leqslant z^{*}, \\
\text { region (2) where } V>z^{*} R, & R \leqslant 1, \text { and } V \leqslant z^{*}, \\
\text { region (3) where } R>1 \text { or } & V>V_{c}=z^{*} .
\end{array}
$$

The GM model provides both the distribution of the relative velocity and the distribution of the radial relative velocities, the latter being needed to compute the collision kernel and differs from the former only in the exponent of the power-law in region (2) (i.e., $D_{2}-d-1$ for the radial counterpart instead of $D_{2}-2 d$ ). The distribution of radial relative velocities can be constructed by projecting the relative velocities in Eq. (1) onto the unit vectors of the corresponding spherical coordinate system and integrating over all angles (see Gustavsson and Mehlig [16] for more details). The model is expected to hold for any Stokes number (i.e., with a finite rate of caustic formation) if two conditions are met:

(1) Particles must sufficiently detach from the flow field (i.e., $V \gg R$ )

(2) The dynamics of the particles have to be insensitive to the nature of the large scale forcing of the system.

Condition 1. defines a Stokes-dependent scale $R^{*}(\mathrm{St})$ below which the power-law due to caustics is observed. With regard to condition 2., for very large Stokes numbers the distribution of relative velocities can be predicted using the variable-range projection model of Gustavsson et al. [24].

It should be noted that the GM model does not explicitly depend on the Kubo number. In isotropic turbulence $\mathrm{Ku} \propto$ $\sqrt{\operatorname{Re}_{\lambda}}$, where $\operatorname{Re}_{\lambda}$ is the Taylor-based Reynolds number, and therefore the GM model does not explicitly depend on the Reynolds number either. The Taylor-based Reynolds number is defined as $\operatorname{Re}_{\lambda}=\frac{u^{\prime} \lambda}{v}$, where the Taylor scale $\lambda=\left(\frac{15 v u^{\prime 2}}{\epsilon}\right)^{1 / 2}$.

The GM model still can have an implicit Reynolds number dependency through the correlation dimension $D_{2}$ or the matching scale $z^{*}$. Previous studies however have shown that the effect of the Reynolds number on the correlation dimension is rather limited $[25,26]$.

\section{NUMERICAL SETUP}

Our aim in this paper is to compute the joint PDF of the relative velocities at small separations of identical inertial particles in turbulent flows using direct numerical simulations (DNS) and compare our results with the predictions of the GM model. Since the GM model does not predict a direct Kubo (or Reynolds) number dependency, we also investigate whether this is justifiable.

Under the assumption of small and heavy particles (i.e., $r \ll \eta$ and $\rho_{p} \gg \rho_{f}$ ), the equations of motion for the particles are given by $[27,28]$ :

$$
\begin{gathered}
\frac{d \mathbf{v}_{i}}{d t}=\frac{\mathbf{u}\left[\mathbf{x}_{i}, t\right]-\mathbf{v}_{i}}{\tau_{p}}, \\
\frac{d \mathbf{x}_{i}}{d t}=\mathbf{v}_{i},
\end{gathered}
$$

where $\mathbf{x}_{i}$ and $\mathbf{v}_{i}$ are the position and velocity vectors of particle $i$ and $\mathbf{u}$ is the velocity of the flow field, governed by the Navier-Stokes equations. The in-house developed DNS we use solves the Navier-Stokes equations using pseudospectral methods. Time integration is performed using a third-order Adams-Bashforth scheme. Both advection and diffusion are treated explicitly, and the $3 / 2$ rule is used to fully deal with aliasing errors (see, e.g., Canuto et al. [29]). Time stepping is restricted by the Courant-Friedrich-Lewy criterion using a Courant number of 0.1 .

The equations of motion of the particles (3) and (4) are integrated using a fourth-order Runge-Kutta scheme. The velocity of the flow field at the particle position is computed using trilinear interpolation. For more details, see Perrin and Jonker [30]. 
TABLE I. Overview of the simulations. Each simulation is shown together with the number of grid points $N_{x}^{3}$, the Taylor-based Reynolds number $\operatorname{Re}_{\lambda}$, the Kubo number $\mathrm{Ku}$, the dimensionless resolution parameter $k_{\max } \eta$, the Stokes numbers St considered, and the number of particles $N_{p}$. The particles are equally distributed among the categories.

\begin{tabular}{lccccccc}
\hline \hline Run & $N_{x}^{3}$ & $\operatorname{Re}_{\lambda}$ & $\mathrm{Ku}$ & $k_{\max } \eta$ & $r(\mu \mathrm{m})$ & $\mathrm{St}$ & $N_{p} / 10^{6}$ \\
\hline S1 & $128^{3}$ & 60 & 3.9 & 3.4 & $10,40,70$ & $0.045,0.72,2.20$ & 0.6 \\
S2 & $256^{3}$ & 105 & 5.2 & 3.2 & $10,40,70$ & $0.050,0.80,2.45$ & 1.2 \\
S3 & $512^{3}$ & 178 & 6.8 & 2.8 & $10,40,70$ & $0.049,0.78,2.39$ & 2.4 \\
\hline \hline
\end{tabular}

In order to construct the PDF of the relative velocities, we use the algorithm of Allen and Tildesley [31] to find neighboring particle pairs (see also Chen et al. [32] and Perrin and Jonker [30]). This algorithm uses cell indexing and linked lists to check only particle pairs which could collide within one time step and therefore avoids the prohibitive $O\left(N_{p}^{2}\right)$ scaling of the computational cost. Using neighboring particle pairs we construct the joint PDF of $V$ and $R$ at the smallest scales. We consider four separation distances $R=10^{-4}, R=$ $10^{-3}, R=10^{-2}$, and $R=10^{-1}$. Results obtained from the DNS have been made nondimensional using the Kolmogorov length scale $\eta$ and the Kolmogorov velocity scale $v_{\eta}=$ $(v\langle\epsilon\rangle)^{1 / 4}$. The DNS allows us to construct $\rho_{\text {DNS }}(V, R)$, from which $\rho(\Delta \mathbf{v}, R)$ can be computed using isotropy: $\rho(\Delta \mathbf{v}, R) \propto$ $\rho_{\text {DNS }}(V, R) / V^{2}$. The phase-space correlation dimension $D_{2}$ has been computed from the spatial correlation dimension $d_{2}$ using $d_{2}=\min \left(D_{2}, d\right)$ [16]. The correlation dimension $d_{2}$ quantifies the spatial dimensionality of the particle distribution and is computed by taking multiple snapshots of the entire particle field and binning all the particle pairs according to their separation distance $R$. Integrating this histogram yields the correlation sum $C(R)$, the number of particle pairs within a separation distance $R$ :

$$
C(R)=\frac{1}{N_{p}^{2}} \sum_{\substack{i, j=1 \\ i \neq j}}^{N_{p}} \mathcal{H}\left(R-\left|\mathbf{x}_{i}-\mathbf{x}_{j}\right|\right) \propto R^{d_{2}}
$$

with $N_{p}$ being the number of particles in the domain, $\mathcal{H}(\cdot)$ the Heaviside function, and $d_{2}$ the correlation dimension.

Three simulations have been performed with different Reynolds numbers. Per simulation three particle categories are considered $(\mathrm{St} \ll 1, \mathrm{St} \approx 1$, and $\mathrm{St}>1$ ) to investigate the validity of the GM model. To ensure that all scales of turbulence are sufficiently resolved and that the particle trajectories are properly tracked, we use a value for the dimensionless resolution parameter $k_{\max } \eta$ of at least 2.8 for all simulations, where $k_{\max }$ is the highest resolvable wave number $\left(N_{x} / 3\right.$ in our case). All dimensionless parameters are shown in Table I.

\section{RESULTS}

Figure 2 shows the distribution of the relative velocities at different separations computed with the DNS and compared with the GM model predictions for a Stokes number around unity. The DNS confirms the three different regimes predicted

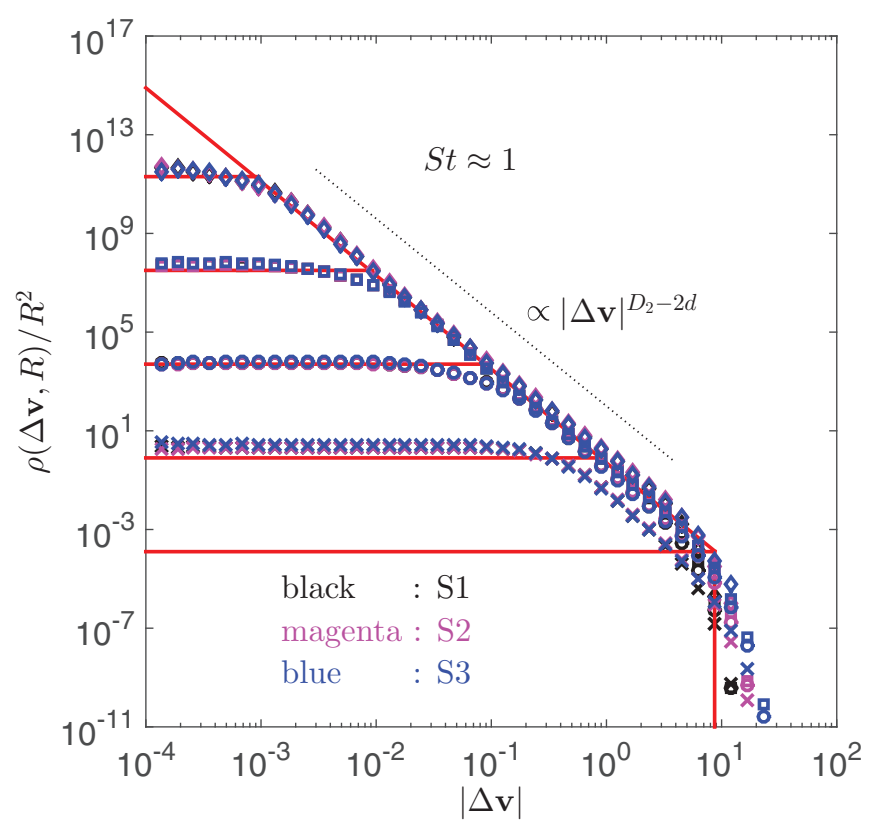

FIG. 2. (Color) The relative velocity distribution for different separations $\left[(\times) R=10^{-1}\right.$, (○) $R=10^{-2},(\square) R=10^{-3}$, and $(\diamond) R=10^{-4}$ ] and different Reynolds numbers [S1 (black), S2 (magenta), S3 (blue)] for the particles with $\mathrm{St} \approx 1$ for which model assumptions 1 . and 2 . are met. The red lines shows the GM model prediction. The dotted line shows the expected power-law based on Eq. (1). See Table I for an overview of the simulations.

by the GM model. In region (1) where pair diffusion is dominant, the plateaus in the distribution of the relative velocities scale according to the predicted $\rho(\Delta \mathbf{v}, R) / R^{2} \propto R^{D_{2}-2 d}$ for all separation distances $R$. In region (2) the distribution behaves universally and collapses onto the single powerlaw $\rho(\Delta \mathbf{v}, R) / R^{2} \propto\left|\Delta \mathbf{v} / z^{*}\right|^{D_{2}-2 d}$. For the highest separation distance considered $(R=0.1)$ the slope of the power law is well predicted but the distribution starts to depend on $R$ which indicates that the universal aspect is lost. As expected the GM model only holds for small separations and $R=0.1$ is on the boundary of its validity. But for practical applications such as atmospheric clouds, a separation distance $R>0.1$ has limited use anyway, since cloud droplets that are large (i.e., $r \approx 100 \mu \mathrm{m}$ ) do not obey linear drag as assumed in Eq. (3); in addition gravity needs to be accounted for. In region (3), the distribution falls off in a manner similar to that of the numerical results of Gustavsson and Mehlig [16].

Although the GM model does not explicitly depend on the Reynolds number, the Reynolds number can influence the model results via both the correlation dimension $D_{2}$ and the matching scale $z^{*}$. In agreement with previous studies $[25,26]$, no significant dependency of the Reynolds number on the correlation dimension is found in Fig. 2. No significant dependency of the Reynolds number on $z^{*}$ is found as well, although the larger the Reynolds number, the better the power-law prediction holds near the boundary with region (3).

Figures 3 and 4 show to what extent the GM model loses its validity if one of the two conditions for the model validity is not met. Figure 3 shows the joint PDF of the relative velocities at different separations for a Stokes number of 


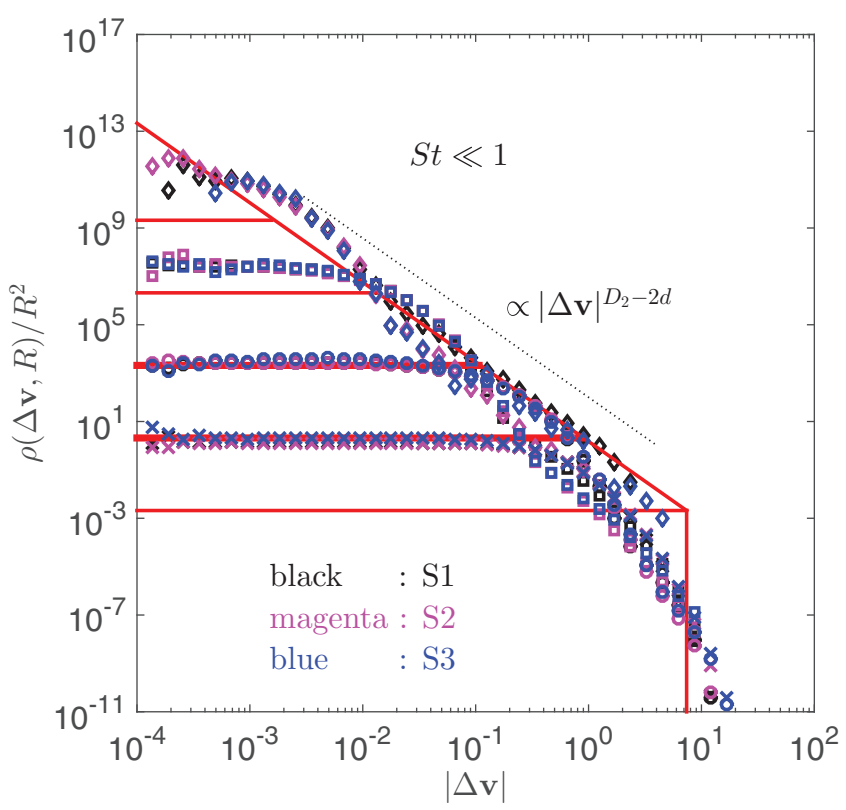

FIG. 3. (Color) Same as Fig. 2 but with St $\ll 1$, which implies that model assumption 1 . is violated. The red lines shows the GM model prediction. The dotted line shows the expected power-law based on Eq. (1). See Table I for an overview of the simulations.

St $\ll 1$. As expected, the model cannot predict the distribution (and especially the power law) since caustics do not sufficiently influence the joint distribution(see assumption 1. requiring that $V \gg R$ ); see Voßkuhle et al. [23] for an estimate of the amount of caustics present for different Stokes numbers.

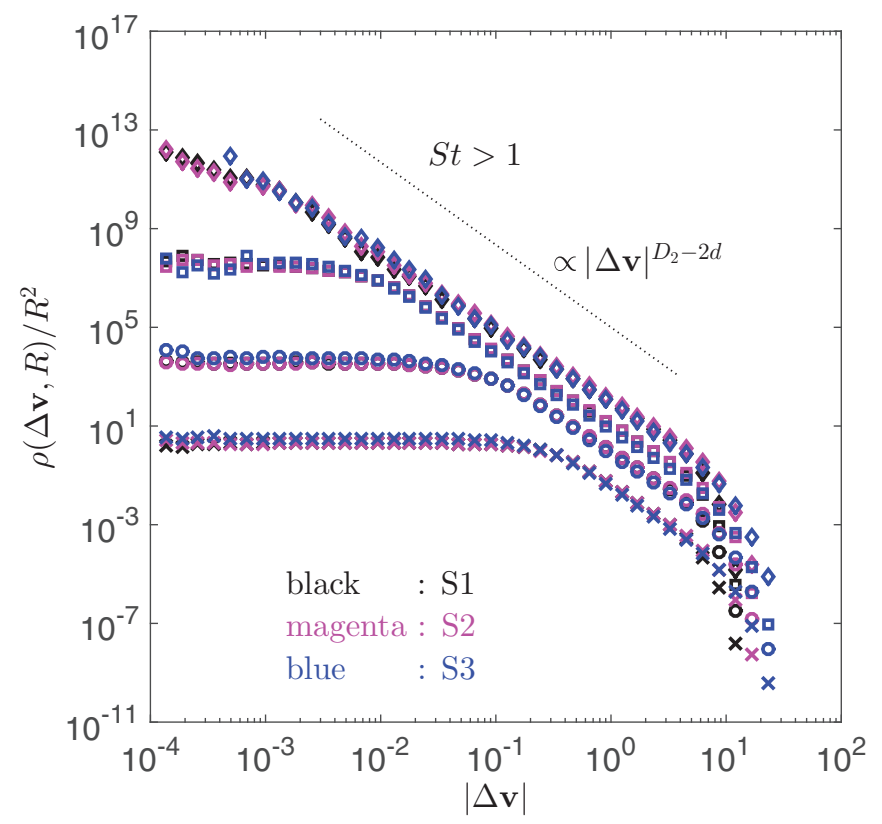

FIG. 4. (Color) Same as Fig. 2 but with St $>1$, which implies that model assumption 2 . is violated. The dotted line shows the expected power-law based on Eq. (1). See Table I for an overview of the simulations.
The particles do not possess enough momentum to be able to sufficiently detach from the flow field and large relative velocities at small separations are very rare. In the limit of a small Stokes number, the theory requires an extremely small separation between particles for the power law to be present since in the power-law region it is imperative that $V \gg R$. Our results support this statement since the slope of the power law seems to be best predicted for the smallest separation distance considered (i.e., $R=10^{-4}$ ). It has to be noted that, in the case that the collision radius, which is defined as the sum of the particle radii, is larger than the required $R^{*}$, sufficiently small separation cannot be physically obtained to observe the power law.

Figure 4 shows the PDF of the relative velocities at different separations for a Stokes number of St $>1$. Although the slope of the power law is fairly accurately predicted, the power law does not collapse onto a single line as in Fig 2. For St $>1$, the particle relaxation time is of the order of the system turnover time, violating assumption 2. that the dynamics of the particles have to be insensitive to the nature of the large scale forcing of the system. As a result, the universal aspect of the power law is lost.

From our previous results we can expect that the GM model can accurately predict the distribution of (radial) relative velocity within the boundaries of its validity discussed above. One could speculate that for higher Reynolds numbers the range of validity of the model in terms of the Stokes number can only improve. For very light particles, the larger the Reynolds number becomes the more extreme events occur in the flow (e.g., Yeung et al. [33]), which promotes the formation of caustics, required by the model. For heavier particles, the larger the Reynolds number becomes the larger the separation between the particle response time and the integral time scale of the system $\left(\propto \operatorname{Re}^{1 / 2}\right)$ becomes, which reduces the impact of the large scale forcings on the particle dynamics.

Note that the results presented here were obtained in the absence of gravity (consistent with the GM model). For some applications, this limits the applicability of the model. Under the effect of gravity, particles can form curtainlike manifolds, which profoundly affect both the spatial distribution and the relative velocity of the particles [12,34-40]). Gravity reduces the formation of caustics [35] and the power-law prediction is expected to fail.

\section{CONCLUSION}

Our key conclusion is that the joint PDF of relative velocities and separations of particles in the absence of gravity in a turbulent flow can be accurately predicted by the model of Gustavsson and Mehlig [16], up to a separation distance of one-tenth of the Kolmogorov scale (dependent on the Reynolds number). This model matches two asymptotic limits of the distribution: one where pair diffusion dominates (i.e., large coherence between particle motion) and one where caustics dominate (i.e., large velocity differences between particles at small separations). The model is expected to hold for any Stokes number if two conditions are met. (i) Sufficient caustics have to be present to allow large velocity differences at sufficiently small separation, otherwise the power-law prediction breaks. This assumption sets a Reynolds-dependent 
scale below which the power law can be observed. (ii) A sufficiently large scale separation has to exist between the particle time scales and the system integral time scales to ensure that the nature of the driving of the system does not influence the dynamics of the particles. No significant Reynolds number effect has been found on the joint PDF of relative velocities, in accordance with the model of Gustavsson and Mehlig [16], although the range of Reynolds numbers studied here is limited. Since the model is capable of accurately predicting the distribution of radial relative velocities at contact, it paves the way to not only a better prediction of the collision kernel but also to a better understanding of the collision characteristics.

\section{ACKNOWLEDGMENTS}

This work is part of the research program of the Stichting Voor Fundamenteel Onderzoek Der Materie (FOM), which is part of the Nederlandse Organisatie voor Wetenschappelijk Onderzoek (NWO). This work was carried out on the Dutch national e-infrastructure with the support of the SURF Foundation.
[1] A. Kolmogorov, Proc. USSR Acad. Sci. 32, 15 (1941).

[2] R. A. Shaw, Annu. Rev. Fluid Mech. 35, 183 (2003).

[3] B. J. Devenish, P. Bartello, J.-L. Brenguier, L. R. Collins, W. W. Grabowski, R. H. A. IJzermans, S. P. Malinowski, M. W. Reeks, J. C. Vassilicos, L.-P. Wang, and Z. Warhaft, Q. J. R. Meteorol. Soc. 138, 1401 (2012).

[4] W. W. Grabowski and L.-P. Wang, Annu. Rev. Fluid Mech. 45, 293 (2013).

[5] L. P. Wang, A. S. Wexler, and Y. Zhou, J. Fluid Mech. 415, 117 (2000).

[6] P. G. Saffman and J. S. Turner, J. Fluid Mech. 1, 16 (1956).

[7] J. Abrahamson, Chem. Eng. Sci. 30, 1371 (1975).

[8] H. J. Voelk, F. C. Jones, G. E. Morfill, and S. Roeser, Astron. Astrophys. 85, 316 (1980).

[9] J. Williams and R. Crane, Int. J. Multiphase Flow 9, 421 (1983).

[10] F. E. Kruis and K. A. Kusters, Chem. Eng. Commun. 158, 201 (1997).

[11] L. I. Zaichik and V. M. Alipchenkov, Phys. Fluids 15, 1776 (2003).

[12] O. Ayala, B. Rosa, and L. P. Wang, New J. Phys. 10, 75016 (2008).

[13] L. Pan and P. Padoan, J. Fluid Mech. 661, 73 (2010).

[14] A. D. Bragg and L. R. Collins, New J. Phys. 16, 055014 (2014).

[15] K. Gustavsson and B. Mehlig, Phys. Rev. E 84, 045304 (2011).

[16] K. Gustavsson and B. Mehlig, J. Turbul. 15, 34 (2014).

[17] M. Wilkinson and B. Mehlig, Europhys. Lett. 71, 186 (2005).

[18] M. Wilkinson, B. Mehlig, and V. Bezuglyy, Phys. Rev. Lett. 97, 048501 (2006).

[19] G. Falkovich, A. Fouxon, and M. G. Stepanov, Nature (London) 419, 151 (2002).

[20] G. Falkovich and A. Pumir, J. Atmos. Sci. 64, 4497 (2007).

[21] G. P. Bewley, E.-W. Saw, and E. Bodenschatz, New J. Phys. 15, 083051 (2013).
[22] L. Ducasse and A. Pumir, Phys. Rev. E 80, 066312 (2009).

[23] M. Voßkuhle, A. Pumir, E. Lévêque, and M. Wilkinson, J. Fluid Mech. 749, 841 (2014).

[24] K. Gustavsson, B. Mehlig, M. Wilkinson, and V. Uski, Phys. Rev. Lett. 101, 174503 (2008).

[25] J. Bec, L. Biferale, M. Cencini, A. Lanotte, S. Musacchio, and F. Toschi, Phys. Rev. Lett. 98, 084502 (2007).

[26] I. Fouxon, Phys. Rev. Lett. 108, 134502 (2012).

[27] M. R. Maxey and J. J. Riley, Phys. Fluids 26, 883 (1983).

[28] R. Gatignol, J. Mec. Theor. Appl. 2, 143 (1983).

[29] C. G. Canuto, M. Y. Hussaini, and A. Quarteroni, Spectral Methods: Evolution to Complex Geometries and Applications to Fluid Dynamics (Springer, New York, 2007).

[30] V. E. Perrin and H. J. J. Jonker, Phys. Rev. E 89, 033005 (2014).

[31] P. Allen and D. J. Tildesley, Computer Simulation of Liquids (Clarendon, Oxford, 1987).

[32] M. Chen, K. Kontomaris, and J. B. McLaughlin, Int. J. Multiphase Flow 24, 1079 (1998).

[33] P. K. Yeung, D. A. Donzis, and K. R. Sreenivasan, J. Fluid Mech. 700, 5 (2012).

[34] E. J. P. Woittiez, H. J. J. Jonker, and L. M. Portela, J. Atmos. Sci. 66, 1926 (2009).

[35] K. Gustavsson, S. Vajedi, and B. Mehlig, Phys. Rev. Lett. 112, 214501 (2014).

[36] Y. Park and C. Lee, Phys. Rev. E 89, 061004 (2014).

[37] J. Bec, H. Homann, and S. S. Ray, Phys. Rev. Lett. 112, 184501 (2014).

[38] L.-P. Wang and M. R. Maxey, J. Fluid Mech. 256, 27 (1993).

[39] O. Ayala, B. Rosa, L. P. Wang, and W. W. Grabowski, New J. Phys. 10, 075015 (2008).

[40] C. N. Franklin, P. A. Vaillancourt, and M. K. Yau, J. Atmos. Sci. 64, 938 (2007). 